\title{
CONFINED ONTIC OPEN SYSTEMS
}

\author{
E. TIEZZI ${ }^{1}$, G. CECCONI ${ }^{2}$ \& N. MARCHETTINI ${ }^{1}$ \\ ${ }^{1}$ Department of Chemistry, University of Siena, Italy. \\ ${ }^{2}$ Consorzio Venezia Nuova, Venezia, Italy.
}

\begin{abstract}
Confined Ontic Open Systems is a new ecodynamic model based on Prigogine's thermodynamics, Ulanowicz's ontic openness and Coherence Domains with defined boundaries and constraints. The model has a wide range of applications, including ecosystems (e.g. invasive species, the lagoon of Venice), ecological economics, urban organization, the supra-molecular structure of water and global biosphere's models. The model is explained in terms of evolutionary thermodynamics and Jørgensen's ecosystems theory, namely "order out of chaos" and eco-exergy. Particular attention is devoted to the emergence of novelties and to the role of water.

Keywords: coherence domains, dissipative structures, open systems.
\end{abstract}

\section{INTRODUCTION}

COOS is an acronym for Confined Ontic Open Systems from the name of the Greek island (Kos, Coo or Coos) where the science of medicine originated at the time of Hippocrates. In Greek mythology, Asclepius, the first great doctor, ascended Mount Olympus as a god and was the first to love water. The primordial element water was considered essential in traditional Asclepiadean therapy, where the ritual of bathing was a form of purification before offering gifts to the great god of health. Hippocrates of Coos separated medicine from religion, magic and philosophy and used it in various fields. He was the first to describe the properties of water as one of the four elements (fire, earth and air) of human nature on which perfection (health, well-being) depends. Health was impaired if one or more of these elements were out of balance. Hippocrates had the merit of describing the power of water and of identifying the origin and characteristics that contribute to knowledge of diseases arising from the environment, as distinct from those of human origin. He not only trusted the religion of the gods, but tried to coexist with it, teaching his students that Nature has its own life, which is not always tied to the supernatural and magic. The major problem of his studies was therefore to give medicine a theoretical instrument of effective explanation and a method of assessment that justified and upgraded the link between theory and experience. The development of the theory was seen as a new approach to medicine that was the forerunner of greater knowledge and the exchange of ideas with doctors operating outside COOS. Hippocrates therefore improved fundamental knowledge that was based on the tradition of religion, magic and observation of natural phenomena [1].

The acronym COOS was coined to describe thermodynamic systems open to interactions with the outside, but that also maintain their own historical identity (ontic), because they are bound to the memory of their origins. The primary aim of this paper is to present COOS as a new thermodynamic model for understanding evolutionary dynamics that create new structures and enable complexity to emerge, increasing the information in a great variety of systems, more commonly known as living systems capable of self-organization. In order to achieve this, living systems need to be protected (confined).

\section{RESULTS AND DISCUSSION}

In order to introduce this new thermodynamic model, it is necessary to distinguish between isolated, closed and open systems. Isolated systems do not exchange energy or matter with their surroundings. Closed systems exchange energy but not matter with the outside. Open systems exchange both energy and matter with their environment. 
The second step is to recognize that some types of open systems have confined ontic openness: they evolve in time and space due to flows of energy and matter across their boundaries that make self-organization possible. Thus a substantial difference distinguishes living from nonliving systems: the former have ontogenetic evolution, whereas the latter can only go towards disorder in time with the exception of some dissipative structures that are capable of a spontaneous generation of complexity, such as into Bénard convection cells and Belousov-Zhabotinsky (BZ) reactions [2, 3].

The COOS model is based on Prigogine's evolutionary thermodynamics and on the idea of ontic openness proposed by Ulanowicz in 2006 [4], revolutionary concepts that challenge the classical thermodynamic principles.

The first law of thermodynamics says that the total energy in the universe, in all its forms, remains unchanged; it can only transform from one form to another in such a way that the total remains constant. This is law of conservation of energy. The second law concerns the use of energy, its availability to do work and its degradation to unusable forms in nature. The thermodynamic function entropy (usually indicated as $S$ ) measures this degree of energy dispersion: the transformations take place spontaneously in the direction of increasing entropy, determining the probabilistic and stochastic succession of events. If we extend this concept to systems that exchange energy and matter with the outside world, two terms of the total change in entropy, $\mathrm{d} S$, are distinguished: $\mathrm{d}_{\mathrm{e}} S$, the transfer of entropy across the boundaries of the system and $\mathrm{d}_{i} S$, the entropy produced within the system by irreversible processes:

$$
\mathrm{d} S=\mathrm{d}_{\mathrm{i}} S+\mathrm{d}_{\mathrm{e}} S \quad \mathrm{~d}_{\mathrm{i}} S \geq 0
$$

Irreversible processes produce entropy. The second law therefore states that irreversible processes lead to a sort of one-way time.

For COOS, it is more useful to refer to Morowitz's equation [5, 6]:

$$
-\mathrm{d} S_{\text {int }}<\mathrm{d} S_{\mathrm{s}}
$$

where $\mathrm{d} S_{\text {int }}$ is the change of entropy for an intermediate system as it is the case for COOS, such that the entropy of COOS may increase but also decrease depending on its capability to self-organize.

Thus a non-equilibrium steady state is generated that must not be confused with thermodynamic equilibrium and in which order may be created from disorder. Order created in this way no longer violates the laws of thermodynamics. Equilibrium is no longer the only attractor of the system, but the system becomes more complex: in fact, it can lose its thermodynamic stability in order to acquire new oscillating states characterized by highly organized structures or chaotic structures (order out of chaos) [7].

For a correct interpretation of processes that characterize the emergence of novelties and self-organization, it is also fundamental to calculate the negative entropy (i.e. order) produced inside open systems and the positive entropy (i.e. disorder) created by them in their environment. The increase in order is only apparent, being at the expense of order in the environment. Overall disorder increases [5].

All living organisms have these thermodynamic properties. They are open systems with their own evolutionary behaviour and the following characteristics:

- they are confined inside a bounded space in which they develop their processes;

- they are ontic, maintaining their internal evolutionary memory that cannot be deleted because it obeys the arrow of time.

COOS may be simple or complex: cells, plants, animals, ecosystems and the planet earth, all systems capable of self-organization, capable of creating order out of chaos $[6,8]$. 
Like all living organisms, pre-biotic systems and physical prototypes of self-organization seem to violate the second principle of thermodynamics: they embody extremely ordered structures that evolve in the direction of increasing order, or decreasing entropy. Emergence of new structures and different organizational levels are related to the presence of liquid water molecules, since these play a fundamental role in the evolution of living systems and generation of chaotic and/or ordered structures. In fact, water is the element that created the fundamental chemical-physical principles for the survival of organisms on planet earth and enabled their ontogenetic development. It has been recognized [9-12] that a simple liquid water molecule self-organizes in extended regions and induces organisms to select and make interactions between different molecular species, causing a high level of complexity inside the system.

Pollack $[13,14]$ showed that there is a thick layer of "special water" (EZ water) at the interface with the walls of a vessel. This water has special properties: it readily donates electrons, so that it is chemically reducing, whereas normal bulk water is oxidizing. There is therefore a higher probability of chemical reactions that give rise to vital processes at the interface between the two layers of water.

A pre-biotic example of a COOS is the BZ-reaction, a class of oscillating chemical reactions [15] in which it is possible to observe the formation of new structures only when there is a suitable equilibrium between water and surface (where the lipids, in this case, have the function of boundaries). Oscillating reactions are the simplest systems manifesting complex behaviour and emerging properties, and they provide insights into the mechanisms of a great variety of biological processes [16]. In order to mimic the biological complexity of a cell environment, we studied the BZ oscillator in the aqueous compartment of different phospholipids/water lamellar phases, since lipids are major components of plasma membranes. The water/lipid system is a good model for the cell environment, since different aqueous domains in living systems are usually separated by a membrane with a single phospholipid bilayer as basic structural unit. Another intriguing result was the disappearance of all BZ patterns and structures when the water phase/lipid ratio was low. In fact, for water concentrations below $70 \% \mathrm{~W} / \mathrm{W}$ with respect to the lipid, no self-organization phenomena were observed. Water is the molecule of life, since all known forms of life and all physiological solutions have a water content of $60-99 \%$ [17, 18].

In nature there are many examples of COOS: let us refer to important features related to birth, as the placenta and the nest. The nests of several birds present the characteristics of COOS because they could be seen as a micro-cosmos protected from the outside dangers but open to exchanges of energy and matter necessary for the development and the survival of young and adult birds. So the nest is the refuge, the house where the birds can grow and organize themselves: it has the same role as home for man, as an ecosystem for the wild-life and as the planet earth for all living organisms. A similar example is the atoll, e.g. the blue hole of J.J. Cousteau in the Caribbean Sea.

The formation of a salt-marsh in the lagoon of Venice was based on this model: the flows of tidal energy and matter on an intertidal surface interface produced compartmentalization that led to the formation of organic matter [19, 20]. Once the first living matter had been established in the form of a microbial mat, the trapping process self-accelerated, giving rise to massive growth of structured biomass and biodiversity. The protective boundaries allowed an increase in halophytic species, developing a biological diversity of plants, birds and fish. A high level of complexity evolved, characterized by a general increment in eco-exergy, an indicator of sustainability that quantifies the quality of energy by measuring the distance of the system from thermodynamic equilibrium. It offers an estimate of the information contained in system structure, because it could increase with increasing biomass and diversity of ecosystem elements [21].

According to the reference [21], eco-exergy is defined by the following equation:

$$
\mathrm{Ex}=T \sum_{i=1}^{i=\mathrm{n}} \beta_{i} \times C_{i}
$$


where $T$ is the absolute temperature, $C_{i}$ is the concentration in the ecosystem of component $i$ (e.g. biomass of a given taxonomic group or functional group), $\beta_{i}$ is a factor able to express roughly the quantity of information embedded in the biomass. Detritus is chosen as reference level, i.e. $\beta_{i}=1$, and exergy in biomass of different types of organisms being expressed in detritus energy equivalents.

In the ancient books, the Terrestrial Paradise (Eden) is also called a confined garden. Thus the concept of confined ontic openness is related to the 'ecological niche', coined by Elton in 1946 [22], which expressed the situation of an organism living with others in a particular environment, involving several types of interaction. The term ecological niche not only includes physical space but also explains the functional role of the species in the biocenosis and its position in the ecosystem. The interactions between the constituents of the system, that are neither isolated nor completely free, determine the beginning of a process of continuous mutual adaptation; all specimens act and react in response to the others in the system [6]. Ecological systems must therefore be open, allowing continuous flow of energy and matter to and from the outside, but they must also have some boundaries, so that species can achieve a high degree of adaptation to the environment and also give rise to new species. Such evolution is related to the idea of confined ontic openness. In fact, ecosystems must also have physical constraints for exchanges with the outside, favouring differences in temperature between source and sink, so they can continue to create order and self-organize.

A case study of an invasive species introduced into a foreign ecosystem was also considered. This phenomenon has been more frequent in recent years because of intercontinental transport of goods and persons. Alien species upset biocenoses, ecological niches and prey-predator models. In this case, the invaded ecosystem is too open: introduction and proliferation of an invasive species upsets native community structure, causing extinctions and reducing biological diversity. Although introductions are not always negative (in some cases they are responsible for an increase in biodiversity), new community structure with different characters may involve loss of genetic heritage stored over decades of evolutionary history. The new ecosystem could be more vulnerable to natural and manmade perturbations because loss of historical identity means loss of some ontic aspects.

The planet earth can also be considered a COOS, since boundaries that regulate the entry and exit of electromagnetic radiation from the troposphere protected and favoured the development of molecules essential for the survival of life and determined all the fundamental cycles that regulate the metabolism of this planetary super-organism [23].

If a thermodynamic system isolates itself, excluding contacts with the external environment, it is destined to degenerate into total disorder. Likewise, if it opens to limitless exchange of energy and matter with the outside, thermodynamic death is again inevitable. Both hypotheses make the evolution of biological, economic, social and regional systems unsustainable [24].

In socio-economic systems, autarchy is based on the principle of self-government and expresses the process by which an isolated system survives on its own resources (not infinite). The system is therefore destined to die. Globalization, the progressive integration of international markets, is interpreted as a similar process leading to erasure of differences (progressive conformity to rules that hold for everyone) and increasing uniformity of systems (loss of information and disappearance of cultural biodiversity). The principles of ecological economics are founded on the openness of the markets but also on some political and economic restrictions, so that there are the preservation of natural resources (that are not limitless) and not only an infinite growth of the man-made capital. This ecological choice for the management of the natural capital could take part in the idea of confined ontic openness.

In a city or social system, the configuration of interactions between the parts, and between the parts and the whole, is the tangible expression of system organization. The parts of the system develop mutual relations, interacting with the others, and exchange information [25]. Also in this case, the idea of COOS can be applied to the creation and civilization of cities that developed for the 
protection provided by their natural boundaries (the lagoon for the city of Venice) or by boundaries constructed by men (medieval city walls) [26].

Another example is the caravanserai in the Arab culture.

It is worthwhile to compare the point of view developed earlier with another that emerged in the last decades from the conceptual background of quantum field theory (QFT). The interplay between these points of view has been recently investigated [27], pointing out that just the extended space-time regions (coherence domains or CDs) produced by the QFT dynamics might assume the properties of the Prigogine's dissipative structures (DSs). This coincidence of the two a priori different concepts of CDs and DSs allows us to define a CDDS dynamics as underlying the COOS formation.

A first example of this coincidence has recently been discussed with regard to the formation of the Belousov-Zhabotinsky system and the role of water in it [28].

Let us now revisit the properties of COOS in the light of QFT.

A CD has a total energy smaller than the corresponding ensemble of noncoherent components. The difference is termed in the physical jargon 'energy gap'.

In the particular case of liquid water it has been shown [29] that the energy gap $\Gamma$ is given by:

$$
\Gamma=h v_{\mathrm{r}}(T)
$$

where $h$ is the Planck constant and $v_{\mathrm{r}}$ is the frequency of the collective oscillation of the CD, which depends on the number of components of the $\mathrm{CD}$ that in turn depends on temperature $T$.

The existence of the energy gap implies that the coherent system should be able to release energy outwards, so that it should be necessarily open. A closed system would be trapped into a noncoherent state, which would instantly relax to the coherent state, when it gets opened. The higher the "energy gap', the more protected the coherent system against external assaults. In normal liquids, for instance, the self-organizing electrodynamic attraction among molecules induced by the coherent dynamics is contrasted by the disruptive role of thermal collisions, so that at each temperature $T$ there is a coherent fraction $F_{\mathrm{c}}(T)$ and a noncoherent fraction $F_{\mathrm{nc}}(T)=1-F_{\mathrm{c}}(T)$. The relative weight of the two factors, and hence the actual value of $\mathrm{F}_{\mathrm{c}}(\mathrm{T})$, is determined by the comparison of the 'energy gap', which protects order, and of the thermal noise $k T$, which enhances disorder. How much the system requires to be confined in order to be protected depends just on the relative weight of 'energy gap' and 'external noise'. For instance, near a wall, water becomes more coherent, as discovered by G. Pollack and his group [13], because the attraction between water and wall increases the 'energy gap' and hence provides a better protection to the coherence of water. Actually in a living organism water is never 'bulk water', because of the huge number of membranes, molecular backbones and interfaces, so that the whole of biological water could be considered interfacial water in the sense of Pollack and therefore much more coherent than normal water.

The 'energy gap' of the CD is the critical parameter to determine how much protected the system is against the external assaults. Since the 'energy gap' cannot be infinite, a coherent system should necessarily have a finite size, whose amount, however, could be increased by deepening the 'energy gap', the actual value of which is determined by the peculiar dynamics governing the system. According to Arani et al. [29] the full size $D$ of the CD is

$$
D=h c / E
$$

where $c$ is the speed of light and $E$ is the amplitude of the energy oscillation. This size could be eroded by the external assaults so that the effective size could be smaller. For instance, the coherence of ions in an electrolytic solution is much higher than that of normal water [30], so that ions are always totally coherent and may form quite large coherence domains. 
The dependence of the energy gap on the system dynamics corresponds to the term 'ontic' in our definition. The time variations of the dynamics, and also its discontinuities in correspondence with phase transitions of the system, are the story of the system, which actually affects, via the energy gap, its tolerance of novelties and its strength.

In a social system, it is always possible to define its optimal size that varies with its internal structure. This size in ancient civilizations was actually smaller than in modern times. A social human organization extended on the whole planet is not possible in the present economic structure, as shown by the crisis of globalization, but could be possible in an entirely different economic structure, where the internal turbulence could be much smaller.

\section{CONCLUSION}

We think that the presence of COOS is the 'conditio sine qua non' for the emergence of novelties. Liquid water molecules favour emergence of novelties and self-organization in pre-biotic systems and all living organisms. With water (the molecule of life) and natural or artificial boundaries, the ontic-openness of the system enables new structures to form, trapping energy inside and simultaneously transforming it from low grade (high entropy) energy into high grade (low entropy) one. Dissipative structures, indeed, maintain their thermodynamic state of non-equilibrium by continuous dissipation of energy outside the system. The disorder produced by the dissipated energy outside the system creates order and self-organization inside. The order produced generates new order and new organization (autocatalytic structures) but if the flow of energy is interrupted or reduced, the structure may collapse and never return to its initial state (irreversibility).

\section{ACKNOWLEDGEMENT}

Authors wish to thank Michela Marchi for her precious work.

\section{REFERENCES}

[1] Moffitt, H.C., Clinicians past, present and future. California State Journal of Medicine, 20(7), pp. 229-231, 1922.

[2] Rossi, F., Lombardo, R., Sciascia, L., Sbriziolo, C. \& Turco Liveri, M.L., Spatio-temporal perturbation of the dynamics of the ferroin catalyzed Belousov-Zhabotinsky reaction in a batch reactor caused by sodium dodecyl sulphate micelles. Journal of Physical Chemistry B, 122, pp. 7244-7250, 2008. doi:10.1021/jp8003739

[3] Marchettini, N. \& Rustici, M., Effect of medium viscosity in a closed unstirred BelousovZhabotinsky system. Chemical Physical Letters, 317(6), pp. 647-651, 2000. doi:10.1016/ S0009-2614(99)01411-6

[4] Ulanowicz, R.E., Ontic closure and the hierarchy of scale. Annals of the New York Academy of Sciences, 901, pp. 266-271, 2006.

[5] Tiezzi, E., The Essence of Time, WIT Press Publications: Southampton and Boston, pp. 1-123, 2003.

[6] Tiezzi E., Steps Towards an Evolutionary Physics, WIT Press Publications: Southampton and Boston, pp. 1-157, 2006.

[7] Prigogine, I. \& Stengers, I., La Nouvelle Alliance. Métamorphose de la Science, Gallimard: Paris, 1979.

[8] Jørgensen, S.E., Fath, B.D., Bastianoni, S., Marques, J.C., Müller, F., Nielsen, S.N., Patten, B.C., Tiezzi, E. \& Ulanowicz, R.E., A New Ecology: Systems Perspective, Elsevier: Amsterdam, pp. 1-288, 2007.

[9] Szent-Györgyi, A., Bioenergetics, Academic Press: New York, USA, pp. 143, 1957. 
[10] Voeikov, V.L., Biological significance of active oxygen-dependent processes in aqueous systems. Water and the cell, eds G. Pollack, D. Cameron, Wheatly Springer Press: NL, pp. 285-298, 2006.

[11] Del Giudice, E. \& Preparata, G., Coherent dynamics in water as a possible explanation of membrane formation. Journal of Biological Physics, 20, pp. 105-116, 1994. doi:10.1007/ BF00700426

[12] Del Giudice, E. \& Preparata, G., A new QED picture of water: understanding a few fascinating phenomena. Macroscopic Quantum Coherence, eds E. Sassaroli, et al., World Scientific: London, UK, pp. 108-129, 1998.

[13] Pollack, G.H., Cells, Gels and Engines of Life. Ebner and Sons: Seattle, USA, pp. 305, 2001.

[14] Zheng, J.M., Chin, W.C., Khijniak, E., Khijniak E. Jr., \& Pollack, G.H., Surfaces and interfacial water: evidence that hydrophilic surfaces have long-range impact. Advances in Colloid and Interface Science, 127, pp. 19-27, and papers quoted therein.

[15] Rossi, F., Ristori, S., Rustici, M., Marchettini, N. \& Tiezzi, E., Dynamics of pattern formation in biometric systems. Journal of Theoretical Biology, 255(4), pp. 404-412, 2008. doi:10.1016/j. jtbi.2008.08.026

[16] Rossi,F.\&TurcoLiveri, M.L.,Chemical self-organization in self-assembling biomimetic systems. Ecological Modelling, 220(16), pp.1857-1864, 2009. doi:10.1016/j.ecolmodel.2009.04.040

[17] Magnani, A., Marchettini, N., Ristori, S., Rossi, C., Rossi, F., Rustici, M., Spalla, O. \& Tiezzi, E., Chemical waves and pattern formation in the 1,2-dipalmitoyl-sn-glycero-3-phosphocholine/ water lamellar system. Journal of American Chemical Society, 126, pp. 11406-11407, 2004. doi:10.1021/ja047030c

[18] Marchettini, N., Ristori, S., Rossi, F. \& Rustici, M., An experimental model for mimicking biological systems: the Belousov-Zhabotinsky reaction in lipid membranes. International Journal of Ecodynamics, 1, pp. 55-63, 2006. doi:10.2495/ECO-V1-N1-55-63

[19] Cecconi, G., Cerasuolo, C. \& Turco, F., Naturalizzazione delle barene artificiali. Accademia Nazionale dei Lincei: La salvaguardia di Venezia e della sua laguna, Roma, Italy, 2008.

[20] Cecconi, G., Cerasuolo, C., Marchettini, N. \& Tiezzi, E., Salt-marshes as emergent novelties in the Venice Lagoon. Ecological Modelling, 220(16), pp. 1870-1873, 2009. doi:10.1016/j. ecolmodel.2009.04.034

[21] Jørgensen, S.E., Exergy. Encyclopedia of Ecology, eds S.E. Jørgensen \& B.D. Fath, Elsevier: Oxford, pp. 1498-1509, 2008. doi:10.1016/B978-008045405-4.00689-3

[22] Elton, C., Competition and the structure of ecological communities. Journal of Animal Ecology, 15(1), pp. 54-68, 1946. doi:10.2307/1625

[23] Tiezzi, E., The End of Time, WIT Press Publications: Southampton and Boston, pp. 1-200, 2003.

[24] Pulselli, F.M., Bastianoni, S., Marchettini, N. \& Tiezzi, E., The Road to Sustainability - GDP and future generations. WIT Press Publications: Southampton and Boston, pp. 1-197, 2008.

[25] Pulselli, R.M. \& Tiezzi, E., City out of Chaos - Urban Self-organization and Sustainability. WIT Press Publications: Southampton and Boston, pp. 1-161, 2009.

[26] Cecconi, G., Cerasuolo, C., Del Giudice, E., Marchettini, N. \& Tiezzi, E., Ecosystem selforganization in the Venice Lagoon. Ecosystems and Sustainable Development VII, eds. C.A. Brebbia \& E. Tiezzi, WIT Press Publications: Southampton and Boston, pp. 35-41, 2009.

[27] Del Giudice, E., Pulselli, R.M. \& Tiezzi, E., Thermodynamics of irreversible processes and quantum field theory: an interplay for the understanding of ecosystem dynamics. Ecological Modelling, 220(16), pp. 1874-1879, 2009. doi:10.1016/j.ecolmodel.2009.04.035 
[28] Marchettini, N., Del Giudice, E., Voeikov, V. \& Tiezzi, E., Water: a medium where dissipative structures are produced by a coherent dynamics. Biophysical Chemistry, submitted.

[29] Arani, R., Bono, I., Del Giudice, E. \& Preparata, G., QED coherence and the thermodynamics of water. International Journal of Modern Physics B, 9, pp. 1813-1841, 1995. doi:10.1142/ S0217979295000744

[30] Del Giudice, E., Preparata, G. \& Fleischmann, M., QED coherence and electrolyte solutions. Journal of Electroanalytical Chemistry, 248(2), pp. 110-116, 2000. doi:10.1016/S0022-0728(00)00019-X 